\title{
Comparing the Impact of Prescribing One Dose or Two Doses of Injecting Betamethasone for Pregnant Women on Preterm Infants Outcomes
}

\author{
Saymari F. ${ }^{1} M D$, Zand Vakili F.* $M D$, Rezaie M. ${ }^{1} M D$, Mansori M. ${ }^{2} M D$, Afkhamzadeh A. ${ }^{3} M D$
}

*Obstetrics \& Gynecology Department, Medicine Faculty, Kurdistan University of Medical Sciences, Sanandaj, Iran

${ }^{1}$ Obstetrics \& Gynecology Department, Medicine Faculty, Kurdistan University of Medical Sciences, Sanandaj, Iran

${ }^{2}$ Pediatrics Department, Medicine Faculty, Kurdistan University of Medical Sciences, Sanandaj, Iran ${ }^{3}$ Family \& Social Medicine Department, Medicine Faculty, Kurdistan University of Medical Sciences, Sanandaj, Iran

\begin{abstract}
Aims: The use of corticosteroids and abandoning the delivery are of preventive actions of newborns' respiratory distress syndrome. The aim of this clinical trial was to compare the effect of one versus two doses of Betamethasone on the outcomes of preterm infants.

Materials \& Methods: In this quasi-experimental clinical trial, 100 preterm labor pregnant women who referred to Sanandaj Be'sat Hospital in 2013 were selected by purposive sampling method and were divided into one dose (12 $\mathrm{mg}$ Betamethasone) and two doses (12mg Betamethasone with the interval of 24 hours) groups. Information about delivery and gender, weight and Apgar scores of newborn were recorded in the checklist. Newborns were examined for infant respiratory distress syndrome, necrotizing enterocolitis, cerebral hemorrhage and neonatal death. Data were analyzed in SPSS 20 software by Chi-square and Mann-Whitney U tests.

Findings: 14 newborns (28\%) in one dose and 13 (24.5\%) in two doses group had respiratory distress syndrome $(p=0.689)$. 4 newborns $(8 \%)$ in one dose and $7(13.2 \%)$ in two doses group had enterocolitis and 8 newborns (16\%) in one dose and $9(17 \%)$ in two doses group needed respiratory support ( $p>0.05)$. Conclusion: Single-dose or two doses administration of Betamethasone in preterm mothers have no impact on newborn after birth.
\end{abstract}

\section{Keywords}

Betamethasone [http://www.ncbi.nlm.nih.gov/mesh/68001623]

Infant, Premature [http://www.ncbi.nlm.nih.gov/mesh/68007234]

Respiratory Distress Syndrome, Newborn

[http://www.ncbi.nlm.nih.gov/mesh/?term=D012127]

\footnotetext{
${ }^{*}$ Corresponding Author

Tel: +988733288119

Fax: +988733288119

Address: Obstetrics \& Gynecology Department, Be'sat Hospital, Mardoukh Junction, Keshavarz Street, Sanandaj, Iran

fr.fzandvakili@gmail.com

Received: December 22, $2014 \quad$ Accepted: March 1, $2015 \quad$ ePublished: April 16, 2015
} 


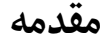

بتامتازون، كورتيكواستروئيدى است كه در باردارىهاى در معرض

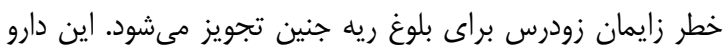

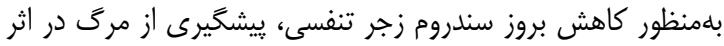

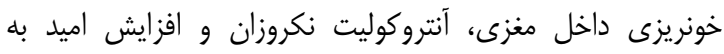
زندگى نوزاد نارس تزريق مى شود [ب-1-1]. كوريوآمنيونيك و

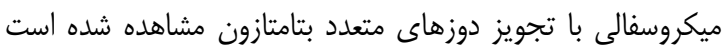

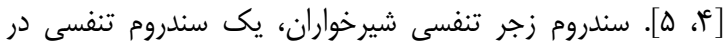

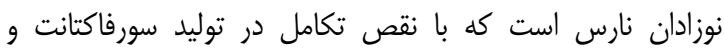

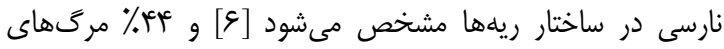

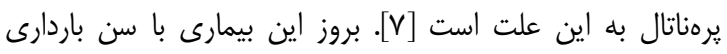

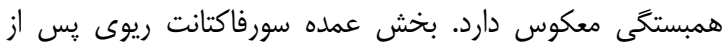

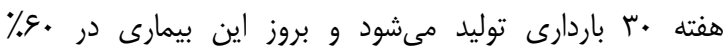

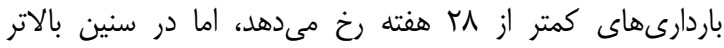

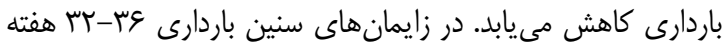

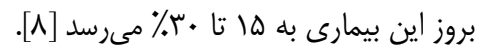

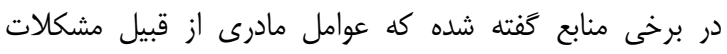

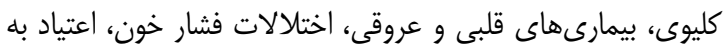

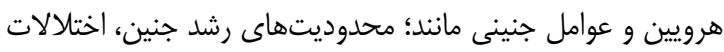

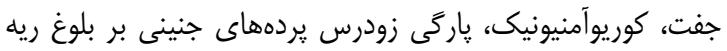

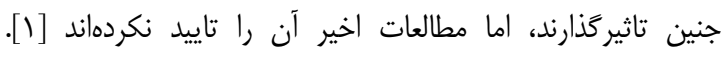

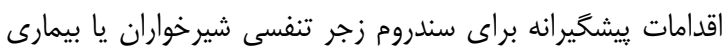

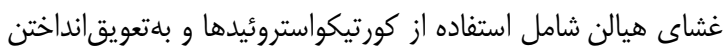

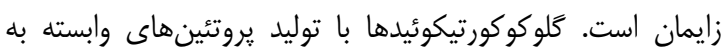

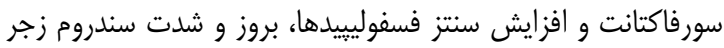

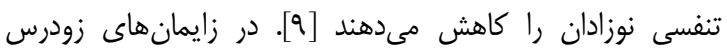

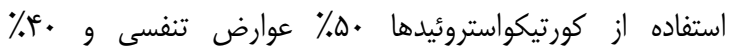

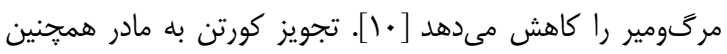

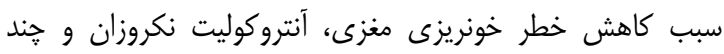

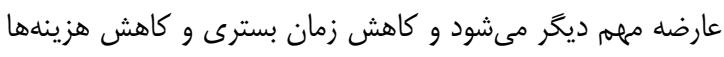

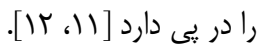

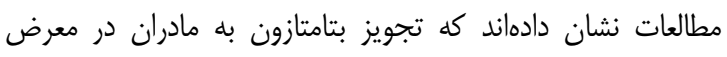

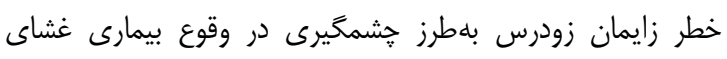

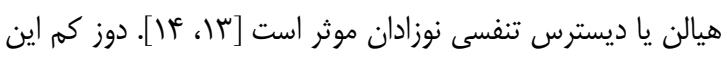

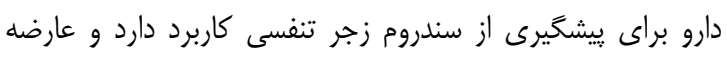

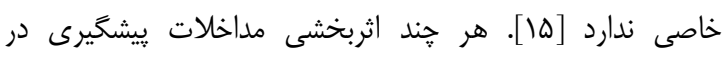

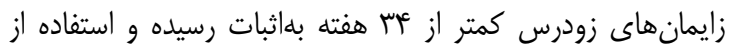

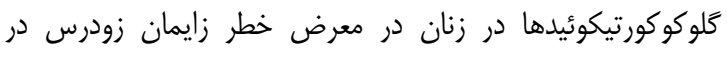

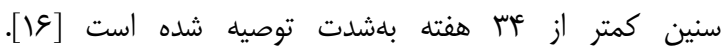

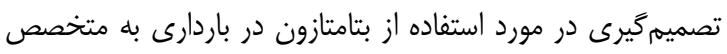

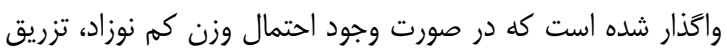

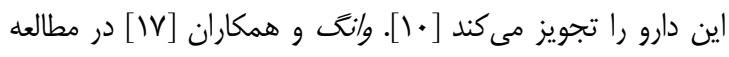

\section{مقايسه تاثير تجويز تك دوز و دو دوز بتامثنازئن تزريقى در زنان باردار بر يِيامدهاى نوزادان زودرس}

فرشته سيمرى MD كروه زنان، دانشكده يزشكى، دانشعاه علوم يزشكى كردستان، سندج، ايران MD فرناز زندوكيلى كروه زنان، دانشكده يزشكى، دانشخاه علوم يزشكى كردستان، سنداج، ايران MD معصومه رضايیى

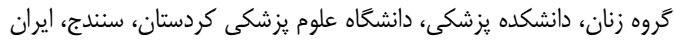
MD مجيد منصورى كَروه كودكان، دانشكده يزشكى، دانشخاه علوم يز شكى كردستان، سنندج، ايران

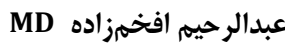

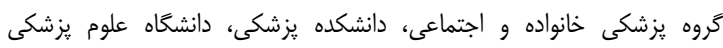
كردستان، سنتدج، ايران

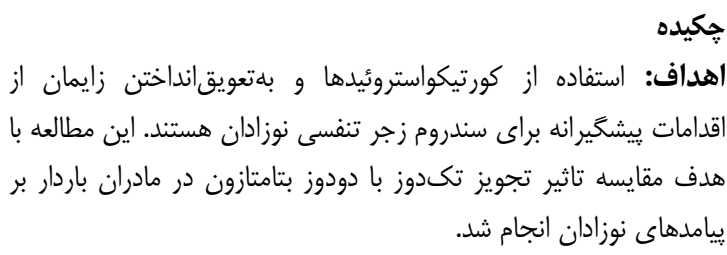

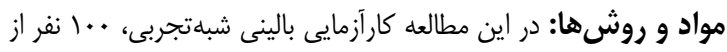

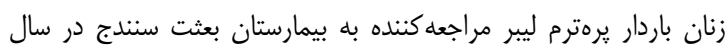

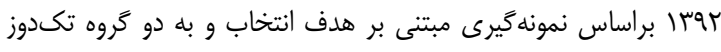

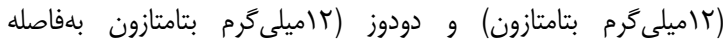

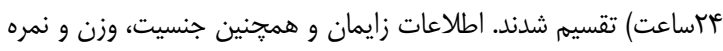

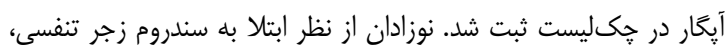

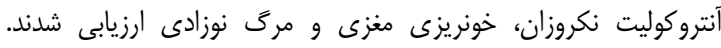

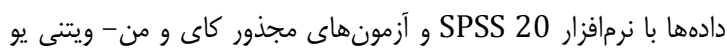

مورد تحليل قرار گرفت.

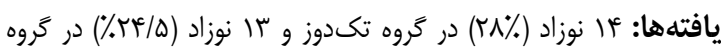

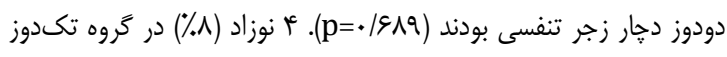

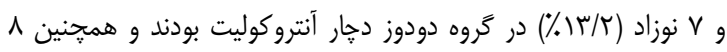

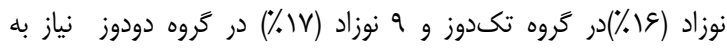

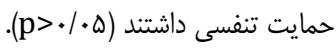

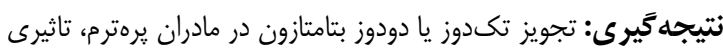
بر وضعيت نوزاد يس از تولد ندارد.

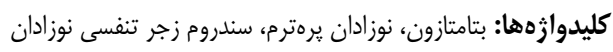

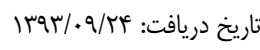

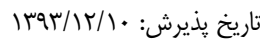

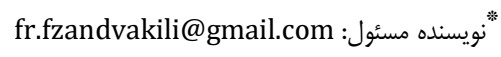

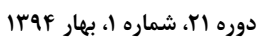

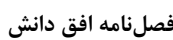




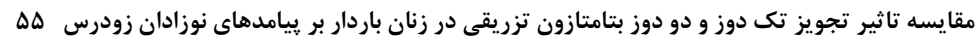

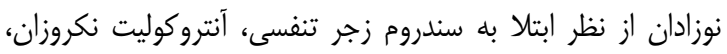
خونريزى مغزى و مرگ نوزادى توسط متخصص نوزادان مورد معاينه قرار گرفتند و ارزيابى ها ثبت مرئ شدري

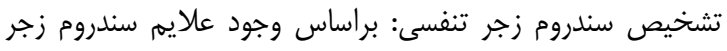

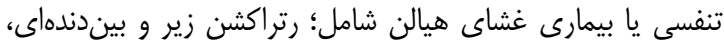

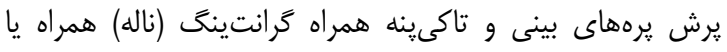

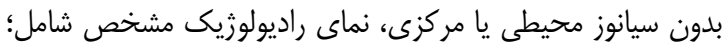

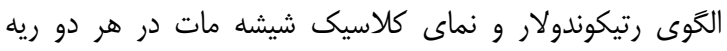

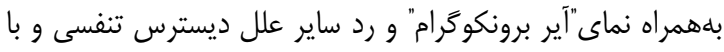

تاييد يزشك فوق تخصص نوزادان بود.

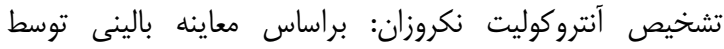

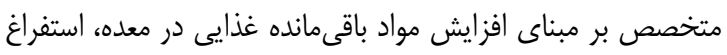

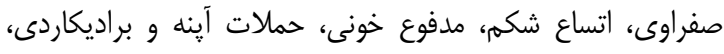

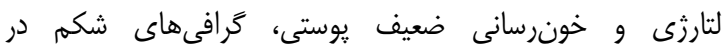
وضعيتهاى خوابيده به يشت و "كراس تيبل لاترال" ابتدا اتساع

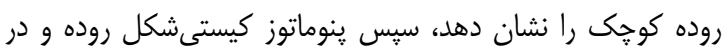
نهايت، گاز درون وريد يورت نمايان شود. تشخيص خونريزى مغزى: براساس معاينه بالينى نوزاد توسط متخصص كودكان تعيين شد. تشخيص مرى نوزادى: براساس نظر متخصص نوزادان در خصوص سنص مرى نوزاد تعيين شد. براى كاهش سوگيرى و كوركردن فرآيند زردآورى دادهها،

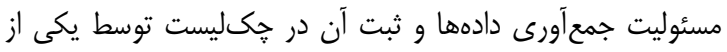

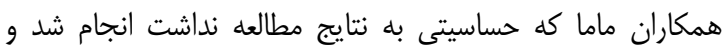

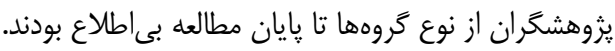

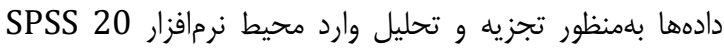

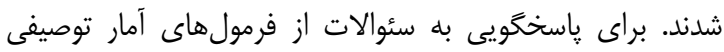

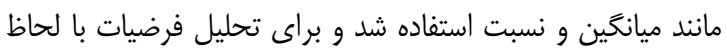

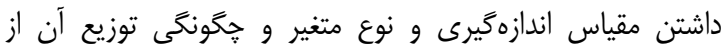

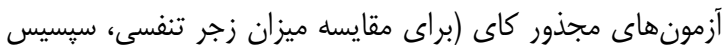

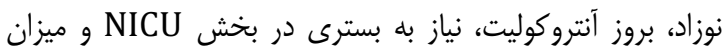

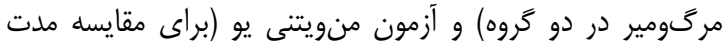

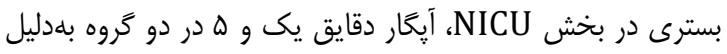

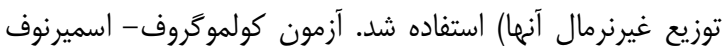
نشان داد كه توزيع متغيرهاى كمى نرمال نبود.

\section{يافتهها}

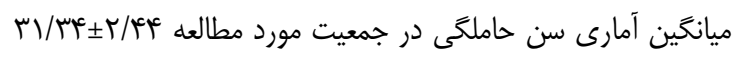

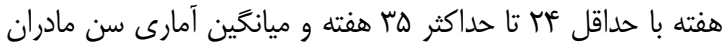

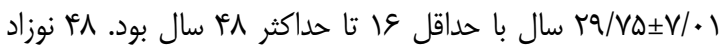

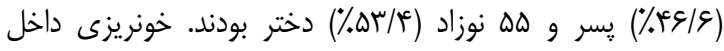

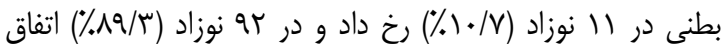

خود عهr نفر از مادران نوزاد نارس را مورد بررسى قرار دادند و نوزادان در سه گروه تكدوز، دودوز و شاهد (بدون تزريق) قرار

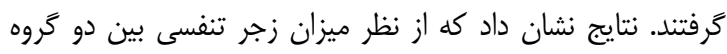

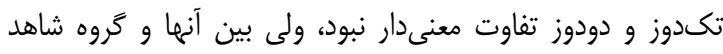

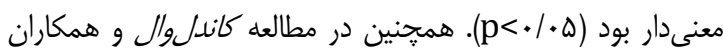

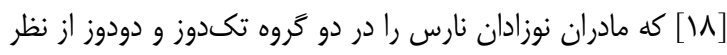

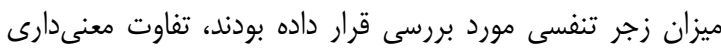

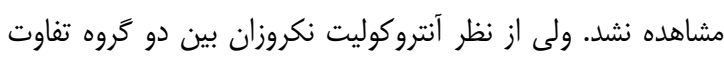
معنى مار بود. با توجه به انجام مطالعات محدود در خصوص مقايسه اثر تكدوز و ود

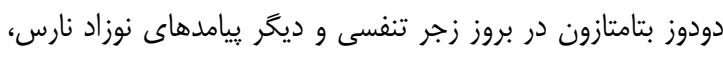

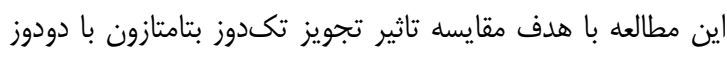
بتامتازون در مادران يرهترم بر ييامدهاى نوزادان انجام شد.

\section{مواد و روشها}

در اين مطالعه كارآزمايى بالينى بلهورت شبهتجربى، تعداد +.. انفر

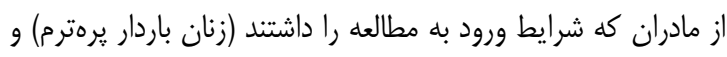

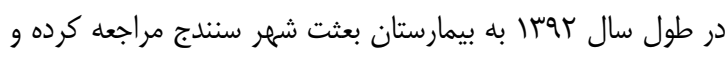

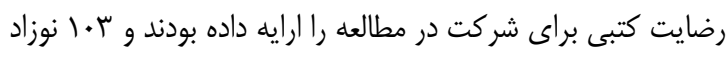

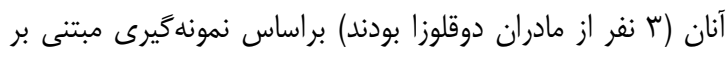

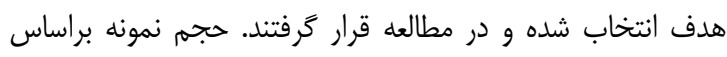

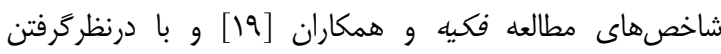

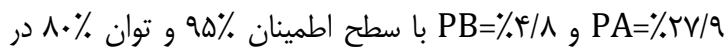

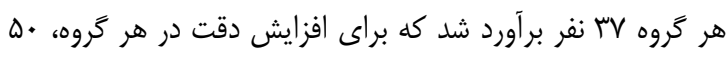
زن باردار و نوزادش مورد بررسى قرار گرَفتند. نمونهها بلهورت غيرتصادفى (بلمنظور رعايت ملاحظات اخلاقى)

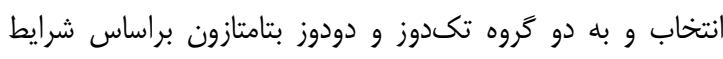

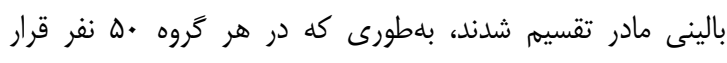

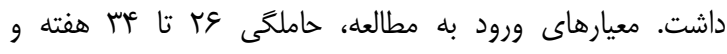
معيارهاى خروج از مطالعه، ابتلاى مادر به دكولمان جفت، مان،

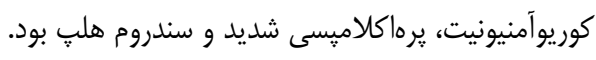

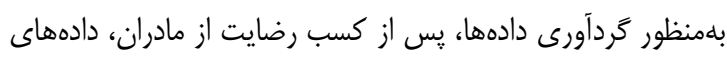

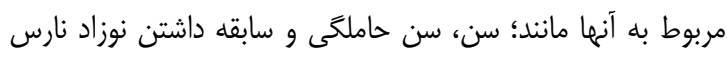

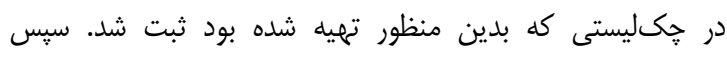

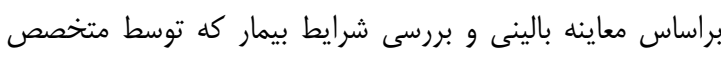

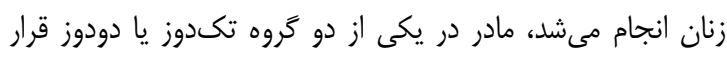

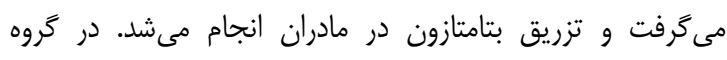
تكدوز rاميلى Tاميلى مادر باردار تزريق شد (روش روتين و استاندارد).

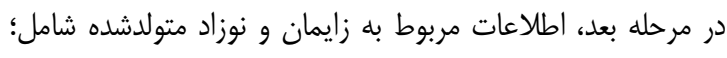

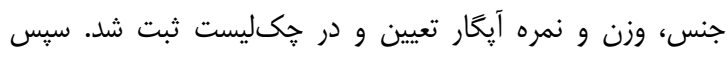


تحت تاثير اين ويزگى ها قرار نكرفته است. قابل توجه است كه در

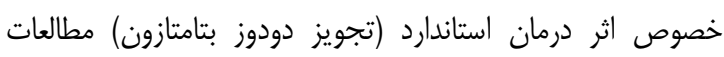

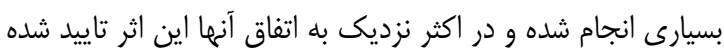

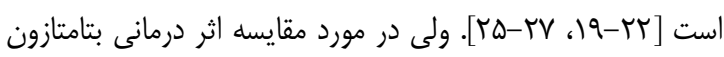

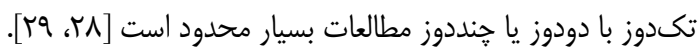

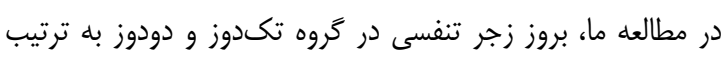

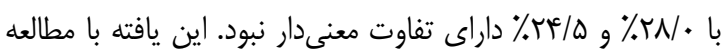

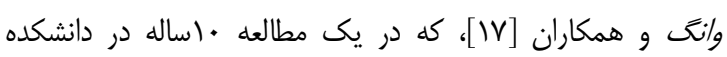

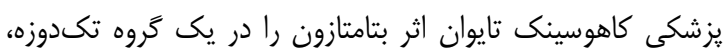

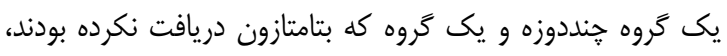

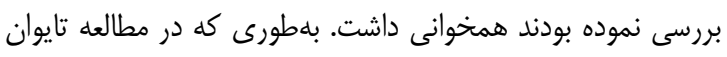

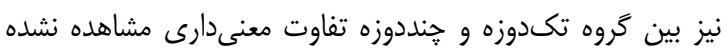

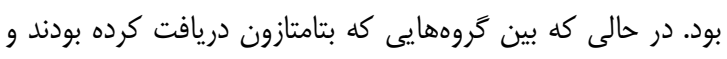

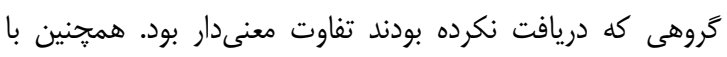

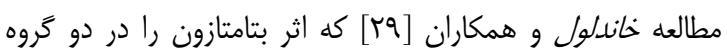

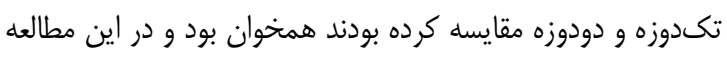

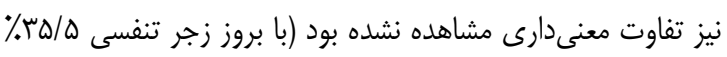

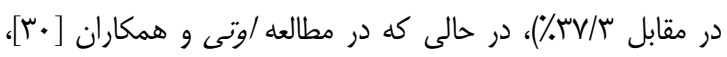

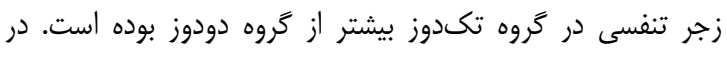

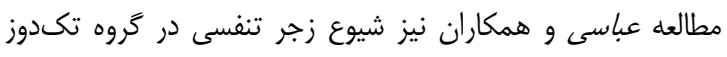

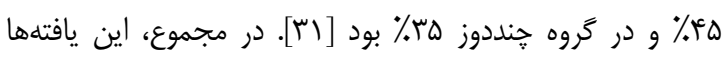

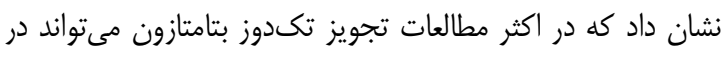

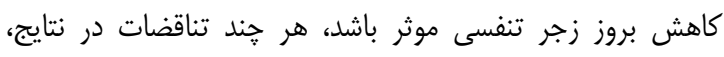

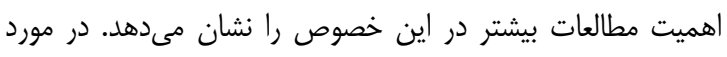

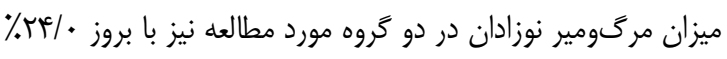

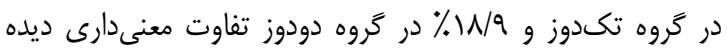

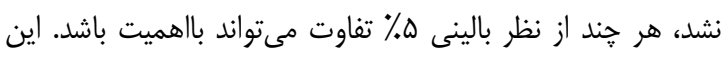

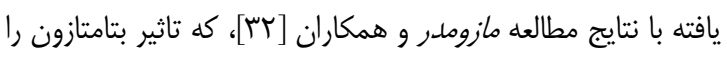

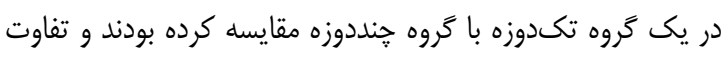

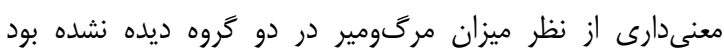

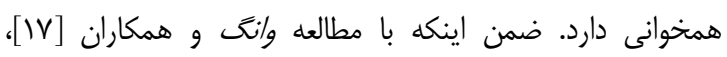

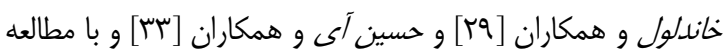

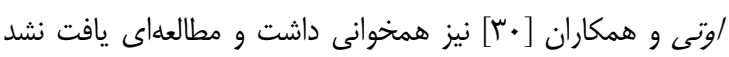

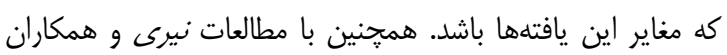

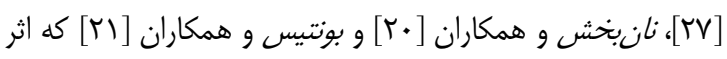

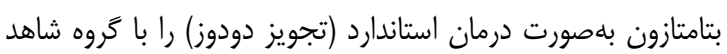

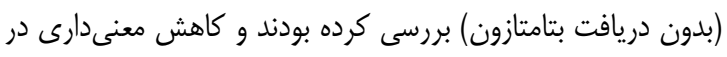

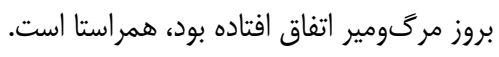

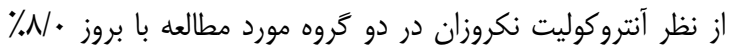

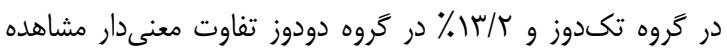

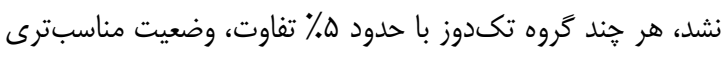
داشت و شايد از نظر بالينى باهميت باشدر باهد اين يافته با مطالعه

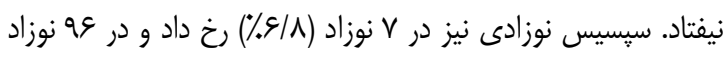

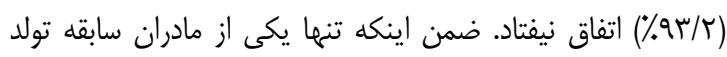

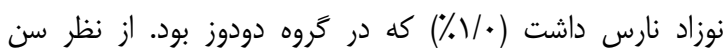

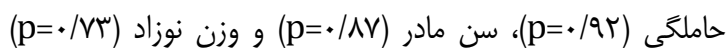

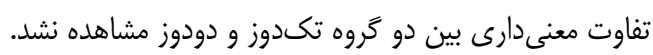

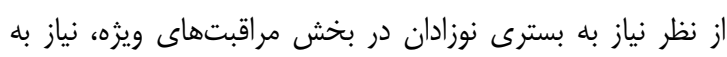

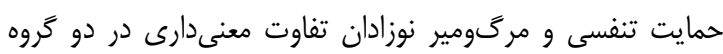

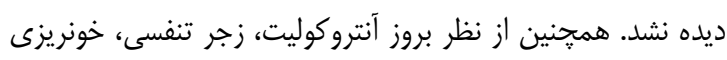

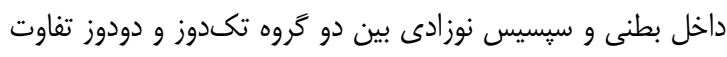

معنى دارى وجود نداشت (جدول (1).

\begin{tabular}{|c|c|c|}
\hline معنى سطحى & تروه دودوز (تعداد=كاه نفر) & تروه تكدوز (تعداد=ه نفر) \\
\hline rat & Vفر (T/r\%) & أنتروكوليت \\
\hline.$/ 819$ & rا نفر (ه/D\%) & زجر تنفسى \\
\hline.$/ 819$ & Vنفر (T/T/F) & 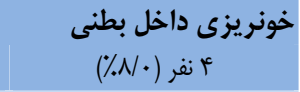 \\
\hline .1 .9 & צ نفر (ك/T/1\%) & سֶيسيس نوزادى \\
\hline & & نياز به بسترى نوز ادان \\
\hline . & أF نفر (FV/F) & 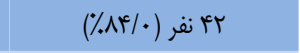 \\
\hline -1/१९ & 9 نفر (•/\%/) & نياز به حمايت تنفسى 1 نفراء/) \\
\hline •/DTA & ا انفر (1\%/9). & 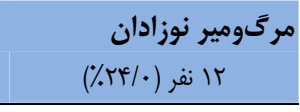 \\
\hline
\end{tabular}

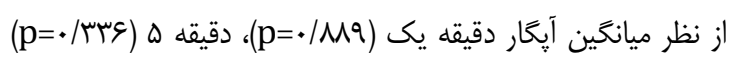

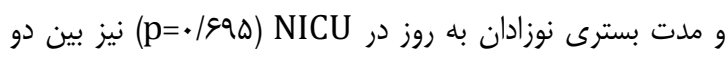
كروه تفاوت معنى دار مشاهده نشد.

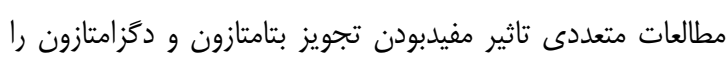

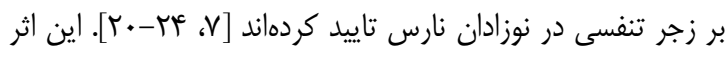

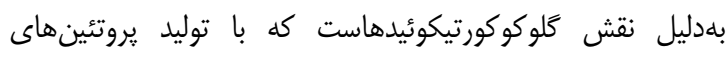

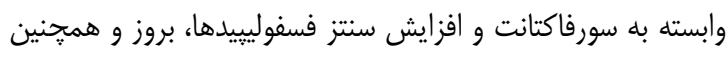

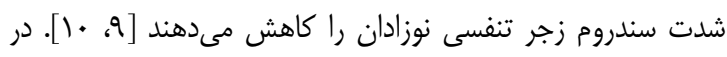

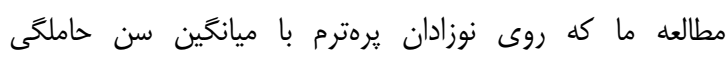

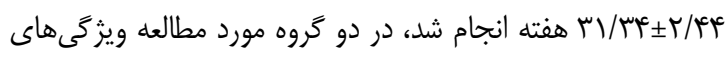

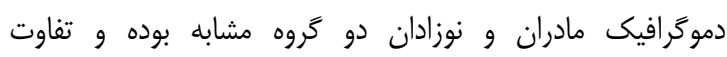
معنى دارى با هم نداشتند. مى توان اظهار داشت نتايج مطالعه حاضر دران 


$$
\begin{aligned}
& \text { بالينى ايران نيز (IRCT2014090912789N6) ثبت شده دون بنان } \\
& \text { است. } \\
& \text { تعارض منافع: هيج گَنه تعارض منافعى توسط نويسندكان بيان } \\
& \text { نشده است. } \\
& \text { منابع مالى: اين مطالعه با حمايت مالى معاونت يخوهشى دانشگاه } \\
& \text { علوم يزشكى كردستان انجام شده است. }
\end{aligned}
$$$$
\text { DV مقايسه تاثير تجويز تك دوز و دو دوز بتامتازون تزريقى در زنان باردار بر ييامدهاى نوزادان زودرس }
$$

\section{منابع}

1- Cunningham F, Leveno K, Bloom S, Hauth J, Rouse D, Spong C. Williams obstetrics. 23 ${ }^{\text {rd }}$ edition. New York: McGraw-Hill Professional; 2009. p. 819.

2- Gamsu HR, Mullinger BM, Donnai P, Dash CH. Antenatal administration of betamethasone to prevent respiratory distress syndrome in preterm infants: Report of a UK multicentre trial. $\mathrm{Br} J$ Obstet Gynaecol. 1989;96(4):401-10.

3- Briggs GG, Freeman RK, Yaffe SJ. Drugs in pregnancy and lactation: A reference guide to fetal and neonatal risk $.4^{\text {th }}$ edition. Baltimore: Williams \& Wilkins; 1994. pp. 8990.

4- Vermillion ST, Soper DE, Newman RB. Neonatal sepsis and death after multiple courses of antenatal betamethasone therapy. Am J Obstet Gynecol. 2000;183(4):810-4.

5- Esplin MS, Fausett MB, Smith S, Oshiro BT, Porter TF, Brancb DW, Varner MW. Multiple courses of antenatal steroids are associated with a delay in long-term Psychomotor development in children with birth weights $<1500$ grams. Am J Obstet Gynecol. 2000;182:S24.

6- Rodriguez RJ, Martin RJ, Fanaroff AA. Respiratory distress syndrome and its management. In: Fanaroff AA, Martin RJ, editors. Neonatal-perinatal medicine: Diseases of the fetus and infant. $8^{\text {th }}$ edition. Mosby; 2006. pp. 1001-11.

7- Meneguel JF, Guinsburg R, Miyoshi MH, de Araujo Peres C, Russo RH, Kopelman BI, et al. Antenatal treatment with corticosteroids for preterm neonates: impact on the incidence of respiratory distress syndrome and intra-hospital mortality. Sao Paulo Med J. 2003;121(2):45-52.

8- Dudell GG, Stoll BJ. Respiratory tract disorders. In: Kliegman RM, Behrman RE, Jenson HB, Stanton BF, editors. Nelson Textbook of Pediatrics. $18^{\text {th }}$ edition. Philadelphia: Saunders; 2007. pp. 728-53.

9- Crowley P. Prophylactic corticosteroids for preterm birth. Cochrane Database Syst Rev. 2000;(2):CD000065.

10- Crowley P, Chalmers I, Keirse MJ. The effects of corticosteroid administration before preterm delivery: An overview of the evidence from controlled trials. Br J Obstet Gynaecol. 1990;97(1):11-25.

11- Asnafei N, Pourreza R, Miri SM. Pregnancy outcome in premature delivery of between 34-37 weeks and the effects of corticosteroid on it. J Gorgan Univ Med Sci. 2004;6(2):57-60. [Persian]

12- Goldenberg RL. The management of preterm labor. Obstet Gynecol. 2002;100(5Pt1):1020-37.

13- Schmitt BD. Instructions for pediatric patients. $2^{\text {nd }}$ edition. Philadelphia: Saunders; 1999.

14- Mansouri M, Seyedolshohadaei F, Company F, Setare Sh, Mazhari S. Effect of antenatal Betamethasone on
خاندلول و همكاران [ra] مغايرت داشت، بلطورى كه در اين

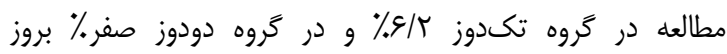
آنتروكوليت نكروزان اتفاق افتاده كه تفاوت نيز معنىدار بوده است.

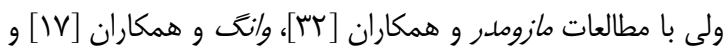

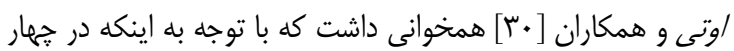

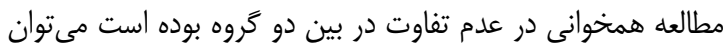
كفت بهاحتمال زياد، تكدوز بتامتازون نيز مىتواند در ييشخيرى از

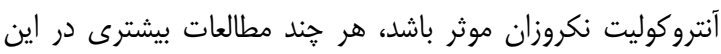

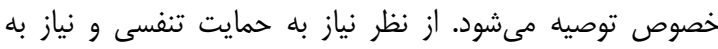

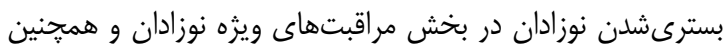

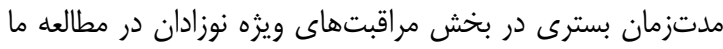

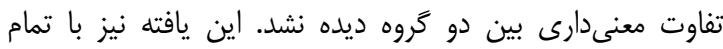

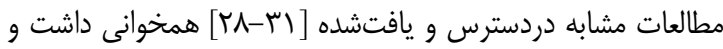

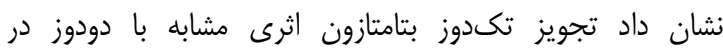

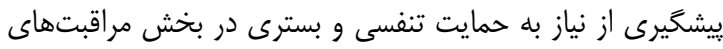

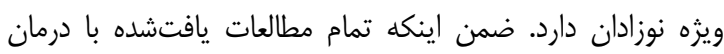
استاندارد بتامتازون نيز موثربودن اين دارو را در تاثير بر كاهش نياز

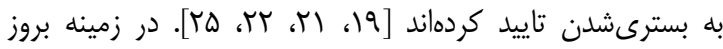

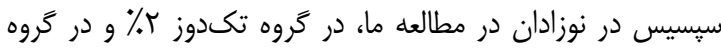

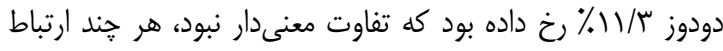

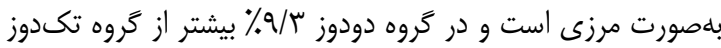

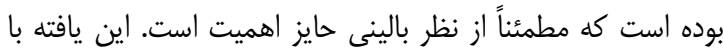

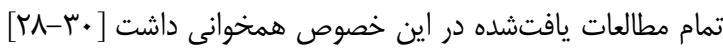

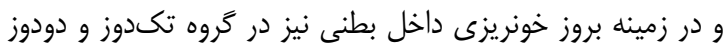

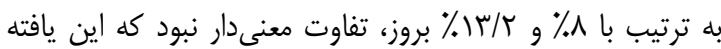

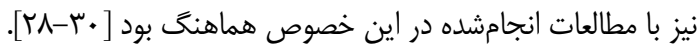

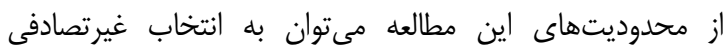

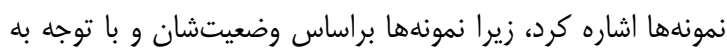

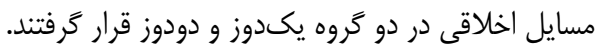

\section{نتيجحه كيرى}

تجويز تكدوز بتامتازون در مادران يرهترم مىتواند بهعنوان

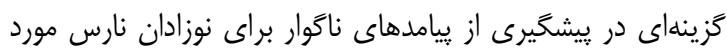

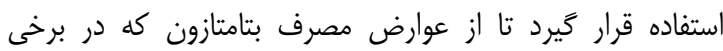

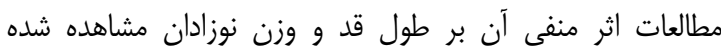
بكاهد، هر هُند براى قضاوت نهايى مطالعات بيشترى ضرورت دات دارد.

تشكر و قدردانى: از اساتيد محترم گروه زنان دانشكده يزشكى

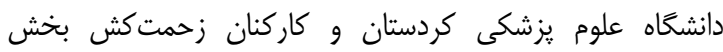
زايمان بيمارستان بعثت سندج تشكر و قدردانى بهعمل مى بـ آيد. تاييديه اخلاقى: انجام اين مطالعه در كميته اخلاق دانشعاه علوم يزشكى كردستان تصويب شده و در سامانه ثبت كارآزمايىهاى 
Am J Obstet Gynecol. 1995;173(1):322-35.

25- Roberts D, Dalziel S. Antenatal corticosteroids for accelerating fetal lung maturation for women at risk of preterm birth. Cochrane Database Syst Rev. 2006; 19(3):CD004454.

26- Moravedji Asl M, Kashanian M, Ahangari Shirzi A. The incidence of respiratory distress syndrome in preterm infants born during the first 24 hours of dexamethasone administration to mothers. Razi J Med Sci. 2005;12(45):173-80. [Persian]

27- Nayeri F, Movaghar-Nezhad K, Assar-Zadegan F. Effects of antenatal steroids on the incidence and severity of respiratory distress syndrome in an Iranian hospital. East Mediterr Health J. 2005;11(4):716-22.

28- Ventolini G, Neiger R, Mathews L, Adragna N, Belcastro $M$. Incidence of respiratory disorders in neonates born between 34 and 36 weeks of gestation following exposure to antenatal corticosteroids between 24 and 34 weeks of gestation. Am J Perinatol. 2008;25(2):79-83.

29- Costa S, Zecca E, De Luca D, De Carolis MP, Romagnoli C. Efficacy of a single dose of antenatal corticosteroids on morbidity and mortality of preterm infants. Eur J Obstet Gynecol Reprod Biol. 2007;131(2):154-7.

30- Peltoniemi OM, Kari MA, Tammela O, Lehtonen L, Marttila R, Halmesmäki E, et al. Randomized trial of a single repeat dose of prenatal betamethasone treatment in imminent preterm birth. Pediatri. 2007;119(2):290-8. 31- Abbasi S, Hirsch D, Davis J, Tolosa J, Stouffer N, Debbs $\mathrm{R}$, et al. Effect of single versus multiple courses of antenatal corticosteroids on maternal and neonatal outcome. Am J Obstet Gynecol. 2000;182(5):1243-9.

32- Mazumder P, Dutta S, Kaur J, Narang A. Single versus multiple courses of antenatal betamethasone and neonatal outcome: A randomized controlled trial. Indian Pediatr. 2008;45(8):661-7.

33- Ay H, Tosun M, Malatyalıoğlu E, Aygün C, Çetinkay MB, Celik H. Comparison of single and double courses of antenatal corticosteroid administration on neonatal mortality and morbidity. J Turkish Ger Gynecol Assoc. 2010;11(1):38-43. prevention of respiratory distress syndrome among neonates with gestational age of 35-36 weeks. J Gorgan Univ Med Sci. 2010;12(3):18-23. [Persian]

15- Dalziel SR, Walker NK, Parag V, Mantell C, Rea HH, Rodgers A, et al. Cardiovascular risk factors after antenatal exposure to betamethasone: 30-year follow-up of a randomised controlled trial. Lancet. 2005;365(9474):1856-62.

16- Effect of corticosteroids for fetal maturation on perinatal outcomes. NIH Consens Statement. 1994;12(2):1-24.

17- Wang YC, Tseng HI, Yang SN, Lu CC, Wu JR, Dai ZK, et al. Effects of antenatal corticosteroids on neonatal outcomes in very-low-birth-weight preterm newborns: A 10 -year retrospective study in a medical center. Pediatr Neonatol. 2012;53(3):178-83.

18- Khandelwal $M$, Chang E, Hansen $C$, Hunter $\mathrm{K}$, Milcarek B. Betamethasone dosing interval: 12 or 24 hours apart? A randomized, noninferiority open trial. Am J Obstet Gynecol. 2010;206(3):e1-11.

19- Fekih M, Chaieb A, Sboui H, Denguezli W, Hidar S, Khairi $H$. Value of prenatal corticotherapy in the prevention of hyaline membrane disease in premature infants. Randomized prospective study. Tunis Med. 2002;80(5):260-5. [French]

20- Nanbakhsh F, Mohadesi H, Ordokhani A, Ghaderi J. Effects of dexamethasone injection on pregnancy outcome in preterm labor. Scientific J Hamadan Nurs Midwifery Fac. 2011;19(2):5-15. [Persian]

21- Bontis N, Vavilis D, Tsolakidis D, Goulis DG, Tzevelekis P, Kellartzis D, et al. Comparison of single versus multiple courses of antenatal betamethasone in patients with threatened preterm labor. Clin Exp Obstet Gynecol. 2011;38(2):165-7.

22- Bonanno C, Wapner RJ. Antenatal corticosteroids in the management of preterm birth: are we back where we started? Obstet Gynecol Clin North Am. 2012;39(1):47-63. 23- Kliegman RM, Stanton BMD, Geme JSt, Schor N, Behrman RE. Nelson textbook of pediatrics. $19^{\text {th }}$ edition. Philadelphia: Saunders; 2011.

24- Crowley PA. Antenatal corticosteroid therapy: A meta-analysis of the randomized trials, 1972 to 1994. 